\title{
Assessing Familiarity of Personnel in an Army-Clinic with New American Heart Association's Basic Life Support (AHA- BLS) 2015 Guideline Updates: A Cross-Sectional Study \& Commentary
}

\author{
Meshkini $\mathrm{M}^{1,2, *}$ \\ ${ }^{1}$ Health-Care Unit, North-West Site of IRI Air Defense Base (Khatam-Al-Anbia), Tabriz, Iran \\ ${ }^{2}$ Road Traffic Injury Research Center, Tabriz University of Medical Sciences, Tabriz, Iran
}

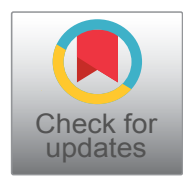

${ }^{*}$ Corresponding author: Meshkini M, Health-Care Unit, North-West Site of IRI Air Defense Base (Khatam-AI-Anbia), Tabriz, Iran

\begin{abstract}
"Despite Accidents, Injuries are predictable and preventable". Cardiovascular events are categorized as common sudden death causes in almost every epidemiological study worldwide, which concerns everybody's preparation and readiness to overcome these issues in the scene of accident. This study was designed as a descriptive cross-sectional one to assess familiarity of an army-clinic personnel with new changes and updates of the latest resuscitation guidelines. For this purpose, a questionnaire was prepared with 14 technical questions and two surveys about personnel assurances with their own and their coworkers' preparedness in the field of accident. Analysis of the returnee data from participants showed weakness and improper familiarity of personnel with new AHA-BLS 2015 guidelines; It's also necessary to be mentioned unfamiliarity seems to be more evident in the seniors' responses, while they were more confident about themselves despite juniors. Which could be a necessary recommendation of Continuous Medical Education (CME) for the personnel. Awareness of Basic Life Support (BLS) and Cardio-Pulmonary Resuscitation (CPR) techniques should be managed as one of the must-to-know topics in any educational and civil defense program, especially in a real continuous method to lower the mortality and morbidity of the accidents by using the power and ability of empowered, knowledgeable and up-todated bystanders.
\end{abstract}

\section{Keywords}

Cardio-Pulmonary Resuscitation (CPR), Continuous Medical Education (CME), Basic Life Support (BLS), Crisis Management, Civil Defense

\section{Introduction}

Preparing for emergency situations and environmental accidents have always been of the most emphasizing policies in I.R. Iran [1]. In-Time action to lower the burden of disease, morbidity and mortality whether primordial and primary preventions to reduce the incidence of accidents and their predisposing situations are recommended to manage the situation from $A$ to $Z$ [2].

Cardiovascular events are found out to be the cause of sudden death in adolescent and elderly age groups in almost every epidemiological study [2-4], familiarity with new and up-to-dated changes of the curriculum and guidelines in the subject is necessary to achieve the prior goals of managing emergency cardiovascular events, whether there are strict rules for medical and emergency staff in some countries that constraint them to be prepared and up-to-dated for the Life Support skills $[3,5,6]$. This study aim was to assess the familiarity of personnel in one of Iranian army-clinics with new advantages and guideline changes of AHA/BLS 2015 and bring commentaries for future studies and programs.

\section{Methods}

This descriptive cross-sectional study was prepared by a questionnaire of 14 technical questions about news in AHA-BLS 2015 guideline familiarity and two surveys of whether people are confident either about their own, or their coworkers' action in the time of crisis (altogether 
Table 1: Questionnaire with answers (translated into English language).

Dear Colleague, this is a questionnaire to assess your familiarity with new changes in Basic Life Support (BLS) v.2015 and assessment of self-esteem and team-preparedness during CPR process. Your accurate answers will help us and any further studies in this field.

1- What's the Best choice for chest compression depth?
A. At least $5 \mathrm{cms}$
B. $5-6.25 \mathrm{cms}$
C. Rigidly $7.5 \mathrm{cms}$
D. As Much as Possible

2- What's the best Airway management maneuver for potential cervical injured patients?
A. Head Tilt-Chin Lift
B. Jaw Thrust

3- How much should a pulse-checking take?
A. Till could be palpable
B. Max 2 seconds
C. Max. 5 Seconds
D. Max. 10 Seconds
4- Where is the best place for adult patients' pulse-checking?
A. Carotid (Neck)
B. Femoral (Groin)
C. Radial (Wrist)
D. Brachial (Elbow)

5- Where is the best place for pediatric patients' pulse-checking?
A. Carotid (Neck)
B. Femoral (Groin)
C. Radial (Wrist)
D. Brachial (Elbow)

6- An Infant has Gasping and pulse rate of 100/min; what should the resuscitator do?

\begin{tabular}{|l|l|l|l|}
\hline $\begin{array}{l}\text { A. Starting CPR with emphasizing } \\
\text { on chest compression prior to } \\
\text { anything else }\end{array}$ & $\begin{array}{l}\text { B. Give Breaths each 5-6 } \\
\text { seconds }\end{array}$ & $\begin{array}{l}\text { C. Give Breaths each 2-5 } \\
\text { seconds }\end{array}$ & D. Do Nothing. \\
\hline
\end{tabular}

7- A pediatric patient doesn't breathe and has a pulse of $50 / \mathrm{min}$, what should the resuscitator do?
A. Starting CPR with emphasizing
B. Give Breaths each 5-6 seconds
C. Give Breaths each 2-5
D. Do Nothing.
on chest compression prior to seconds

anything else

8- A 50 Y/O male patient chocked food while dinner-time. What should the bystanders do?
A. Struck on His back
B. Do nothing till he collapses
C. Press on his abdomen
D. Press on his chest and then start CPR

9- A 10 month baby got something in her throat, what would you do?
A. Alternatively struck on her back
B. Try to pull out the foreign
C. Press on her abdomen
D. Start CPR and chest object by your finger

10- When attempt to remove blockage of foreign throat objects would be stopped?
A. When the foreign object comes out
B. When the patient collapses
C. When the patient's breathing would come back to normal

11- A 10 month baby got something in her throat while eating and suddenly collapses, what would you do?
A. Alternatively struck on her back
B. Try to pull out the foreign
C. Press on her abdomen
D. Start CPR and chest object by your finger

12- What's the best Compression: Breathing ratio in adults?
A. $5: 3$
B. $10: 2$
C. $15: 2$
D. $30: 2$

13- What's the best next step when not finding pulse in an unconscious patient?
A. Starting CPR
B. Give 2 breathes
C. Ask for help

14- What's the best next step when a regular pulse found in an unconscious patient?
A. Starting CPR
B. Assessing Airways
C. Nothing to do

15- How would you assess yourself at all about knowledge, skill, preparedness \& etc. while a CPR event?
A. Best - 5
B. Good - 4
C. Moderately - 3
4. Weak - 4
5. Very feeble

15- How would you assess other colleagues at all about their knowledge, skill, preparedness \& etc. while a CPR event?
A. Best - 5
B. Good - 4
C. Moderately - 3
4. Weak - 4
5. Very feeble

16 questions). They were copied in 25 paper-sheets and distributed among main personnel (seniors) and on-duty personnel (juniors) of the North-West Site of IRI Air Defense Base (Khatam-Al-Anbia) army-clinic of health-care unit. The questionnaire was in Persian language; The English-translated format and answers are demonstrated in Table 1 of this study [3,7]. The returnee papers were almost 17 out of 25, and analysis using GNU-PSPPire v.0.7.9 software been conducted into these categories: 1-Score out of 14, 2-Self Assessment, 3-Coworkers' Assessment, 4-Occupational degree as Seniors and Juniors [8]. Data statistically analyzed in quantitative form for category 1- Exam score, quantitative-qualitative for categories 2 and 3- The Survey questions, and qualitative for category 4-Occupational degree. The Confidence Interval $(\mathrm{Cl})$ was $95 \%$ in central variables assessment of quantitative data analysis. If possible, qualitative data changed into and analyzed as proper quantitative ones; if not they were assessed in their own categories. Due to this study's strict and low sample-size, most of results reported in their raw-descriptive format and not been analyzed statistically . If they would be needed, it could be coordinated with inspection and safety unit of the North-West Site of IRI Air Defense Base (Khatam-Al-Anbia) to been achieved.

\section{Results}

Out of 17 returnee results showed a mean technical score of 6.76 (out of 14); The maximum score was 9 which was achieved by 4 people $(23.53 \%)$ and minimum was 3 , achieved by one (5.88\%). Figure 1 Demonstrates a histogram of answering scores. 


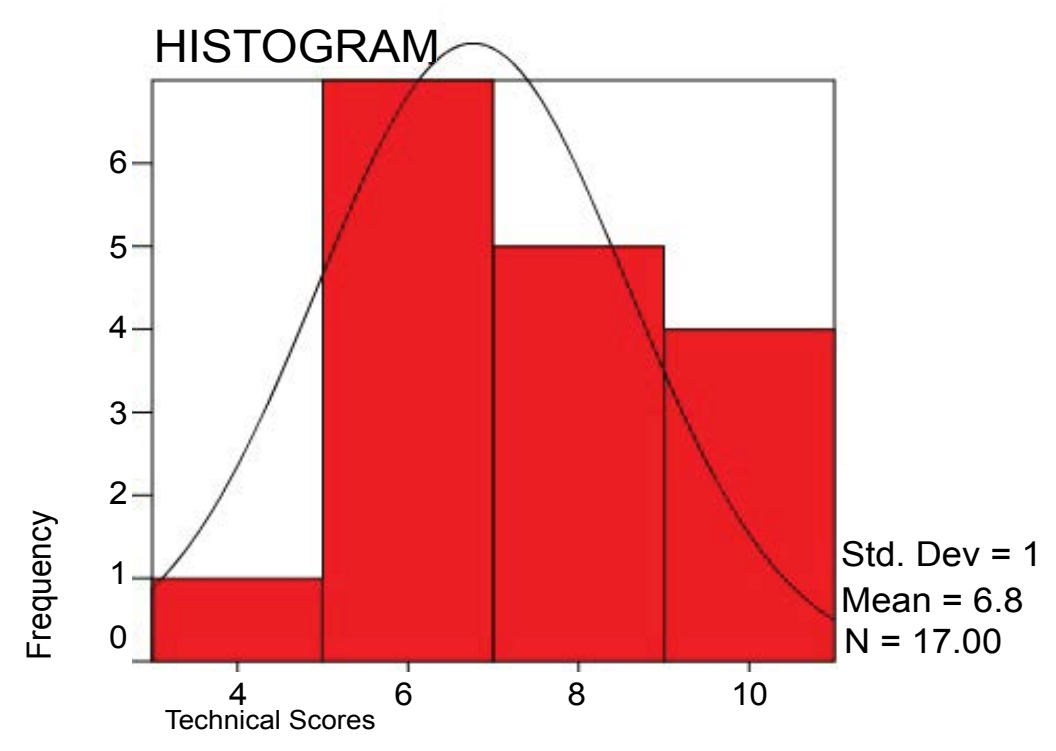

Figure 1: Histogram of Technical Scores.

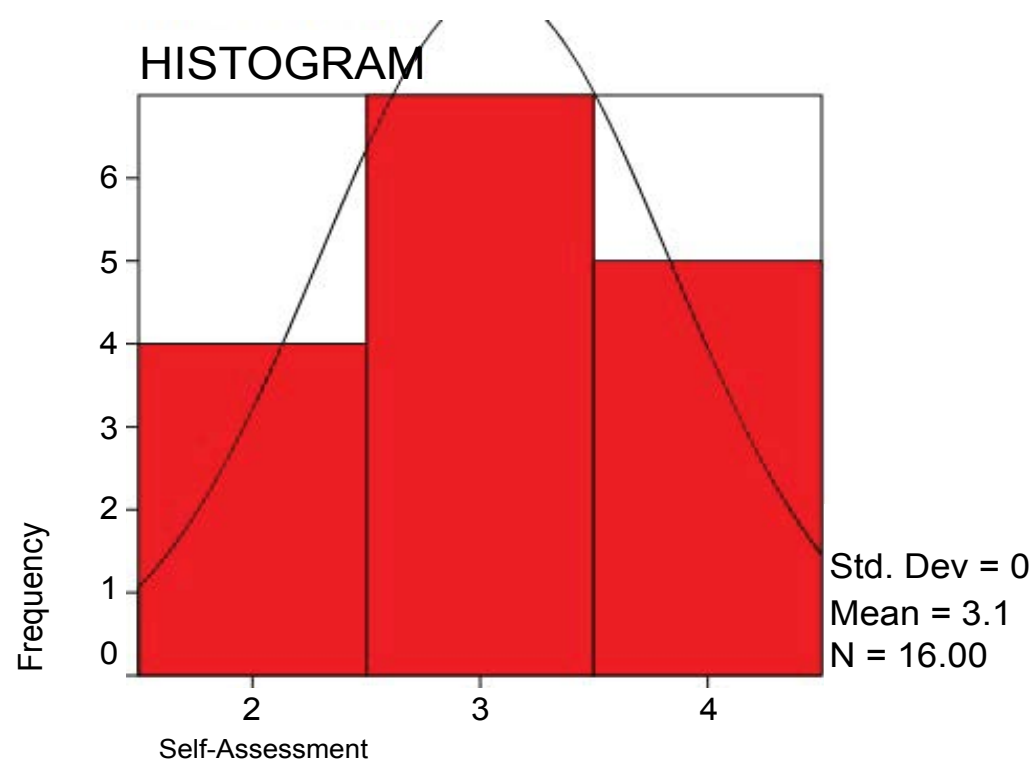

Figure 2: Self-Assessment Histogram.

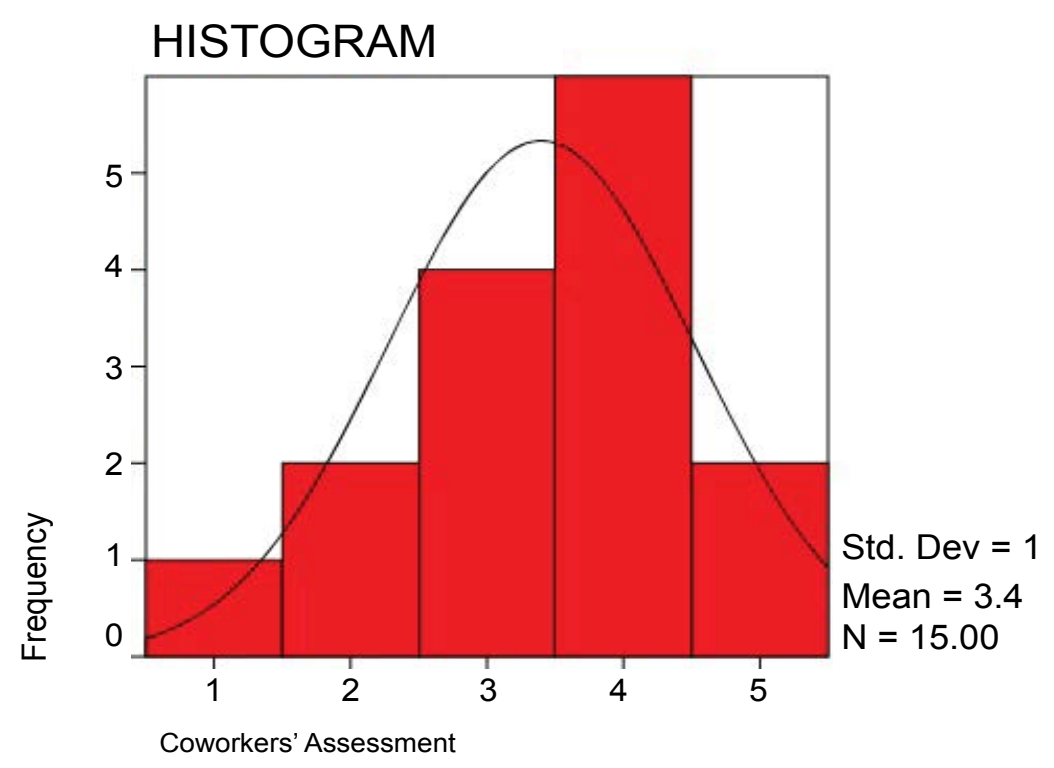

Figure 3: Coworkers' Assessment Histogram. 
In the self-assessment survey, except one who didn't answer the question, analyzing other 16 data showed medium (3/5) confident of 7 people $(41.18 \%)$, following by high (4/5) in 5 people (29.41\%) and low (2/5) of 4 people $(23.53 \%)$ respectively. There was neither veryhigh (5/5) nor very-low (1/5) self-assessment among participants, which at all bring a mean of 3.06/5. Figure 2 demonstrates a histogram of self-assessment scores.

There were 2 unanswered coworkers' assessment survey, however, 15 others assessed their coworkers confident and expertise as high (4/5) in 6 choices (35.29\%), medium (3/5) in 4 choices $(23.53 \%)$, either very-high (5/5) and low (2/5) of each of them by 2 choices $(11.76 \%)$ and very-low(1/5) in one choice (5.88\%), that shows a mean of $3.4 / 5$. Figure 3 demonstrates a histogram of coworkers' assessment scores.

In a comparative comparison of test-score with self-assessment, test-score with coworkers' assessment and self-assessment with coworkers' assessment using Sig. Two-tailed test ( $p$-value $<0.05)$ obtained results were $0.09,0.17$ and 0.55 respectively; that show a true null-hypothesis $(\mathrm{HO})$ of any correlation between staff's technical familiarity either with their self-assessment or coworkers' assessment. However, it seems better correlation in their familiarity-score and self-confident rather than other assessments. Table 2, Table 3 and Table 4 demonstrate results of this section in an integrated detail.

Of those 15-returnee replied paper-sheets 6 were for on-duty personnel and 9 for main personnel. The paper with technical score of 3 who didn't answer the survey questions was of an on-duty staff, while the other paper with technical score of 9 which didn't answer the survey of coworkers' assessment was of a main staff. Excluding these two papers may result in biases of the oncoming results: However, the new analysis showed a mean of $8.33(\min 7, \operatorname{Max} 9)$ of technical questions in on-duty personnel in comparison to 5.89 ( $\min 5$, Max 8 ) in main personnel. Including the previously excluded data in these analysis changes the mean of on-duty to 7.57 and main to 6.20 ; that could be a result of: 1- Up-todated educations in university, red-crescent/cross voluntary program engagement of these (on-duty) personnel and while their 2 months army-educational program, 2- Specialized requirement of on-duty staff (Physicians, Pharmacists, Nurses, Emergency Medical Technicians (EMTs), Red-Crescent/Cross Volunteers) in comparison to main staff (Official Administrative, LAB technicians, and others than main physicians and nursery staff) which could be another selection bias of this study.

Also, on-duty personnel self-assessment and coworkers' assessment mean scores were 2.33/5 and $3.44 / 5$ respectively; the results of main personnel were $3 / 5$ and $3.67 / 5$ respectively. It's to been reported that juniors chose "medium (3/5)" and "low(2/5)" for themselves, also chose "very-high(5/5)" and "very-low(1/5)" for others; while seniors chose "high(4/5)" and "medium(3/5)" for themselves, also "very-high(5/5)" and "medium(3/5)" for others. This could be a result of main staff's higher occupational experience in comparison to on-duty staff.

Mistaken choices' analysis in detail showed the following: Unfamiliarity with the spirit of team-work philosophy in CPR and BLS; "Time is Myocardium" motto; and "CAB is rather than $A B C$ ". That's to say only one chose the seeking help prior to starting CPR (question-13); or only four chose the accurate time for pulse assessment. The best pulse assessing site in adults was chosen correctly by 10 people, while only one answered the question correctly in children. The best answer was for question-2 and Jaw-Thrust maneuver which was chosen by 13 people.

Table 2: Comparative Comparison of Self-Assessment and Coworkers' Assessment.

\begin{tabular}{|c|c|c|c|}
\hline Self-Assessment & Coworkers' Ass & & Total \\
\hline & Very-Low \& Low & Medium, High \& Very-High & \\
\hline Very-Low \& Low & $1(6.7 \%)$ & $3(20 \%)$ & $4(26.7 \%)$ \\
\hline Medium, High \& Very-High & $2(13.3 \%)$ & $9(60 \%)$ & $11(63.3 \%)$ \\
\hline Total & $3(20 \%)$ & $12(80 \%)$ & $15(100 \%)$ \\
\hline
\end{tabular}

Table 3: Comparative Comparison of Self-Assessment and Technical Score.

\begin{tabular}{|l|l|l|l|l|l|l|l|}
\hline Self-Assessment & \multicolumn{3}{|l}{ Technical Score } & \multicolumn{3}{c|}{ Total } \\
\hline & 5 & 6 & 7 & 8 & 9 & \\
\hline Very-Low \& Low & - & - & $1(6.3 \%)$ & $2(12.5 \%)$ & $1(6.3 \%)$ & $\mathbf{4}$ & $(\mathbf{2 5 \% )}$ \\
\hline Medium, High \& Very-High & $4(25 \%)$ & $3(18.3 \%)$ & $1(6.3 \%)$ & $1(6.3 \%)$ & $3(18.3 \%)$ & 12 & $(75 \%)$ \\
\hline Total & $\mathbf{4 ( 2 5 \% )}$ & $\mathbf{3 ( 1 8 . 3 \% )}$ & $\mathbf{2 ( 1 2 . 5 \% )}$ & $\mathbf{3 ( 1 8 . 3 \% )}$ & $\mathbf{4 ( 2 5 \% )}$ & $\mathbf{1 6}$ & $\mathbf{( 1 0 0 \% )}$ \\
\hline
\end{tabular}

Table 4: Comparative Comparison of Coworkers' Assessment and Technical Score.

\begin{tabular}{|c|c|c|c|c|c|c|c|}
\hline \multirow[t]{2}{*}{ Coworkers' Assessment } & \multirow[b]{2}{*}{5} & \multicolumn{4}{|c|}{ Technical Score } & & \multirow[t]{2}{*}{ Total } \\
\hline & & 6 & 7 & 8 & 9 & & \\
\hline Very-Low \& Low & - & - & $1(6.7 \%)$ & - & $2(13.3 \%)$ & 3 & $(20 \%)$ \\
\hline Medium, High \& Very-High & $4(26.7 \%)$ & $3(20 \%)$ & $1(6.7 \%)$ & $3(20 \%)$ & $1(6.7 \%)$ & 12 & $(80 \%)$ \\
\hline Total & $4(26.7 \%)$ & $3(20 \%)$ & $2(13.3 \%)$ & $3(20 \%)$ & $3(20 \%)$ & 15 & $(100 \%)$ \\
\hline
\end{tabular}




\section{Discussion}

This study results are compatible with previous studies and recommendations on continuous education of personnel and making up-to-dated well-skilled staff to overcome emergency events and crisis as well $[3,5,6,9,10]$. These could be designed as CME courses for main personnel, improving and organizing well-established courses for all on-duty staff especially in their 2 months educational period. Also, these Life Support skills should be part of students' educational program while they are at school or university also in a continuous method with latest updates familiarity scope $[3,11,12]$. As well introducing and encouraging the whole community to take part in voluntary courses of Red-Crescent/ Cross (e.g. KHADEM and JAHED projects in Iran) could be an engineering method of life-long empowered bystanders and community, and compatible with civil defense goals [13]. For achieving this purpose, media can play a very large role [14]. American Heart Association (AHA) established web page and YouTube! Channel for simplifying its guidelines for all $[15,16]$ Defining Beetle-Group's "Stayin' Alive" music rhythm as similar CPR chest compression rhythm (about 100 pulses in minute), made it a background music of many TV-series even though there were not in the field of medical dramas $[17,18]$.

\section{Conclusion}

Being prepared against probable accidents and crisis should be a necessary must to know and to do for all in the community. Continuous Education and Assessment of Health-Care providers, encouraging community to take part in these courses (using the capabilities of media and NGOs) could be a good management of crisis resolution $[1,3,19]$. This study was performed in a very low sample-sized community; the larger studies with better randomization and blinding methods are recommended to assess the accuracy of these results.

\section{Acknowledgments}

The Author has to appreciate all whom take part in this study for their kind support and patience. Colonel of HQ, D. Pouresmail, and Lieutenant Colonel Dr. M. Aghai-Shahsavari whom provide the optimal and supportive field to perform this study. Also Dr. Mohammad Zakaria Pezeshki, Associated Professor of Social and Community Medicine's Department of Tabriz University of Medical Sciences-Faculty of Medicine for his tremendous comments and guidance through statistical analysis of study data.

\section{Conflicts of Interest}

The Author had been an Instructor Potential (IP) while taking part in AHA-BLS/ACLS courses held in February 2016, Cornerstone College of Int. Studies, Sharjah, UAE. Also, He was physician-on-duty of health-care unit's personnel at the time of preparing study.

\section{References}

1. https://www.isna.ir/news/94112113721/

2. https://scholar.google.com/scholar?hl=en\&as_ $\mathrm{sdt}=0 \% 2 \mathrm{C} 5 \& \mathrm{q}=$ Epidemiology+and+Control+of $+\mathrm{Com}-$ mon+Disordersin+Iran\&btnG=\%20the $\% 202001 \% 20$ book

3. Neumar RW, Shuster M, Callaway CW, Gent LM, Atkins DL, et al. (2015) Part 1: Executive Summary: 2015 American Heart Association Guidelines Update for Cardiopulmonary Resuscitation and Emergency Cardiovascular Care. Circulation 132: S315-S367.

4. Glantz SA, Parmley WW (1991) Passive smoking and heart disease. Epidemiology, physiology, and biochemistry. Circulation 83: 1-12.

5. Morrison LJ, Gent LM, Lang E, Nunnally ME, Parker MJ, et al. (2015) Part 2: Evidence Evaluation and Management of Conflicts of Interest: 2015 American Heart Association Guidelines Update for Cardiopulmonary Resuscitation and Emergency Cardiovascular Care. Circulation 132: S368-S382.

6. Mancini ME, Diekema DS, Hoadley TA, Kadlec KD, Leveille MH, et al. (2015) Part 3: Ethical Issues: 2015 American Heart Association Guidelines Update for Cardiopulmonary Resuscitation and Emergency Cardiovascular Care. Circulation132: S383-S396.

7. Kleinman ME, Brennan EE, Goldberger ZD, Swor RA, Terry M, et al. (2015) Part 5: Adult Basic Life Support and Cardiopulmonary Resuscitation Quality: 2015 American Heart Association Guidelines Update for Cardiopulmonary Resuscitation and Emergency Cardiovascular Care. Circulation 132: S414-S435.

8. GNU-PSPP.

9. Adib Hajbagheri M, Afazel M R, Mousavi S G A, Noorizad $S$ (2001) Evaluation of knowledge and skills of medical personnels of Kashan hospitals regarding cardiopulmonary resuscitation. Feyz 5: 96-103.

10. Jaberi Y, Changizian L, Mazlomzadeh S (2011) Predictors of Outcome in In-Hospital Cardio-Pulmonary Resuscitation. ZUMS Journal 19: 48-57.

11. Singletary EM, Charlton NP, Epstein JL, Ferguson JD, Jensen JL, et al. (2015) Part 15: First Aid: 2015 American Heart Association and American Red Cross Guidelines Update for First Aid. Circulation 132: S574-S589.

12. American College of Surgeons (2012) Advanced trauma life support: ATLS; student course manual. 9edn. Chicago, III: American College of Surgeons, 366.

13. http://khadem.ir/

14. http://farsi.khamenei.ir/speech-content?id=2338

15. American Heart Association | American Stroke Association.

16. American Heart Association - Youtube!

17. Stayin'Alive | Wikipedia.

18. ACLS.com. Basic Life Support \& CPR for Adults.

19. http://farsi.khamenei.ir/speech-content?id=3383 